\title{
GLOBAL EXISTENCE AND BLOW UP OF SOLUTIONS FOR A COMPLETELY COUPLED FUJITA TYPE SYSTEM OF REACTION-DIFFUSION EQUATIONS
}

Abstract. We examine the parabolic system of three equations

$$
\begin{aligned}
& u_{t}-\Delta u=v^{p}, \\
& v_{t}-\Delta v=w^{q}, \quad x \in \mathbb{R}^{N}, t>0, \\
& w_{t}-\Delta w=u^{r},
\end{aligned}
$$

with $p, q, r$ positive numbers, $N \geq 1$, and nonnegative, bounded continuous initial values. We obtain global existence and blow up unconditionally (that is, for any initial data). We prove that if $p q r \leq 1$ then any solution is global; when $p q r>1$ and $\max (\alpha, \beta, \gamma) \geq N / 2$ (where $\alpha, \beta, \gamma$ are defined in terms of $p, q, r)$ then every nontrivial solution exhibits a finite blow up time.

1. Introduction and main results. In this paper we consider the system

$$
\begin{aligned}
u_{t}-\Delta u & =v^{p}, \\
v_{t}-\Delta v & =w^{q}, \\
w_{t}-\Delta w & =u^{r},
\end{aligned}
$$

for $t>0, x \in \mathbb{R}^{N}$ with $N \geq 1, p, q, r>0$ and

$$
\begin{aligned}
& u(0, x)=u_{0}(x), \\
& v(0, x)=v_{0}(x), \quad x \in \mathbb{R}^{N}, \\
& w(0, x)=w_{0}(x),
\end{aligned}
$$

where $u_{0}, v_{0}, w_{0}$ are nonnegative, continuous, bounded functions.

Let us recall that the system (1.1)-(1.2) has a nonnegative classical solution in $S_{T}=[0, T) \times \mathbb{R}^{N}$ for some $T>0$ (cf. for instance a related

1991 Mathematics Subject Classification: 35B30, 35K55, 35K57.

Key words and phrases: blow up, global existence, reaction-diffusion system. 
argument in $[\mathrm{EH}]$ ). Our primary concern is to describe two cases: $T=\infty$, when the system has bounded solutions for any $S_{t}, t>0$ (global solutions), and $T<\infty$, when solutions are unbounded beyond $T$ (they blow up in a finite time $T$ ). In this paper, we discuss these cases in terms of $p, q, r$ and $N$ only. Some additional dependence on the initial data $u_{0}, v_{0}, w_{0}$ (which implies that both situations coexist) will be considered in another paper.

The Cauchy problem

$$
\begin{array}{ll}
u_{t}-\Delta u=u^{p}, & t>0, x \in \mathbb{R}^{N}, \\
u(0, x)=u_{0}(x), & x \in \mathbb{R}^{N},
\end{array}
$$

has been analyzed by several authors (see [F1], [F2]).

Also the system of two reaction-diffusion equations has been dealt with in case of coupled systems.

For instance, in $[\mathrm{EH}]$ and $[\mathrm{AHV}]$ global existence and blow up results were discussed for the problem

$$
\begin{aligned}
& u_{t}-\Delta u=v^{p}, \quad(x, t) \in \mathbb{R}^{N} \times(0, T), \\
& v_{t}-\Delta v=u^{q}, \quad(x, t) \in \mathbb{R}^{N} \times(0, T), \\
& u(0, x)=u_{0}(x) \geq 0, \\
& v(0, x)=v_{0}(x) \geq 0,
\end{aligned}
$$

while in [EL] a more general system was studied:

$$
\begin{array}{ll}
u_{t}-\Delta u=u^{p_{1}} v^{q_{1}}, & (x, t) \in \mathbb{R}^{N} \times(0, T), \\
v_{t}-\Delta v=u^{p_{2}} v^{q_{2}}, \quad(x, t) \in \mathbb{R}^{N} \times(0, T), \\
u(0, x)=u_{0}(x) \geq 0, \\
v(0, x)=v_{0}(x) \geq 0 .
\end{array}
$$

Our goal is to extend Fujita type global existence-nonexistence theorems to systems of three equations.

Let

$$
A=\left[\begin{array}{lll}
0 & p & 0 \\
0 & 0 & q \\
r & 0 & 0
\end{array}\right] .
$$

We denote by $(\alpha, \beta, \gamma)^{t}$ the solution of $(A-I) X=(1,1,1)^{t}$. We can easily find that

$$
\alpha=\frac{1+p+p q}{p q r-1}, \quad \beta=\frac{1+q+q r}{p q r-1}, \quad \gamma=\frac{1+r+r p}{p q r-1},
$$

where

$$
\operatorname{det}(A-I)=p q r-1 .
$$

We formulate 
Theorem 1. Suppose $\operatorname{det}(A-I) \leq 0$. Then every solution of $(1.1)-(1.2)$ is global.

Theorem 2. Suppose $\operatorname{det}(A-I)>0$. If $\max (\alpha, \beta, \gamma) \geq N / 2$ then (1.1)-(1.2) never has nontrivial global solutions.

We prove Theorem 1 in Section 3; Theorem 2 is proved by contradiction in Section 4. Section 2 contains some auxiliary tools.

2. Preliminaries. As we have mentioned, solutions of (1.1)-(1.2) are classical in some $S_{T}$ (that is, $(u, v, w)(x, t) \in C^{2,1}\left(\mathbb{R}^{N} \times(0, T)\right)$ ). Such solutions satisfy the formulas

$$
\begin{aligned}
& u(t)=S(t) u_{0}+\int_{0}^{t} S(t-s) v(s)^{p} d s, \\
& v(t)=S(t) v_{0}+\int_{0}^{t} S(t-s) w(s)^{q} d s, \\
& w(t)=S(t) w_{0}+\int_{0}^{t} S(t-s) u(s)^{r} d s,
\end{aligned}
$$

where $S(t)$ is an operator semigroup and $S(t) \xi_{0}$ is the unique solution of $\xi_{t}-\Delta \xi=0, \xi(0)=\xi_{0}(x)$, where

$$
S(t) \xi_{0}(x)=\int_{\mathbb{R}^{N}}(4 \pi t)^{-N / 2} \exp \left(-\frac{|x-y|^{2}}{4 t}\right) \xi_{0}(y) d y .
$$

REMARK 2.1. If $(u, v, w)$ is a nontrivial solution of (1.1)-(1.2) on $[0, T]$, then there exists $t_{0} \in(0, T)$ such that $u(x, \tau)>0, v(x, \tau)>0$ and $w(x, \tau)>0$ for $(x, \tau) \in \mathbb{R}^{N} \times\left(t_{0}, T\right)$.

Proof. Let $\left(x_{i}, t_{i}\right), i=1,2,3$, be such that $u\left(x_{1}, t_{1}\right)>0, v\left(x_{2}, t_{2}\right)>0$ and $w\left(x_{3}, t_{3}\right)>0$. Let $t_{0}=\max \left(t_{1}, t_{2}, t_{3}\right)$. From formula $(2.1)_{1}$ for $\tau \in$ $\left(t_{1}, T-t_{1}\right)$

$$
\begin{aligned}
u(\tau) & =S\left(\tau-t_{1}\right) u\left(t_{1}\right)+\int_{0}^{\tau-t_{1}} S\left(\tau-t_{1}-\eta\right) v(\eta)^{q} d \eta \\
& \geq S\left(\tau-t_{1}\right) u\left(t_{1}\right),
\end{aligned}
$$

and since $S(\tau)>0$ we get $u(\tau)>0$ on $\mathbb{R}^{N}$ for $\tau>t_{1}$. Similarly, $v(\tau)>0$ on $\mathbb{R}^{N}$ for $\tau>t_{2}$ and $w(\tau)>0$ on $\mathbb{R}^{N}$ for $\tau>t_{3}$.

We also need 
LEMma 2.1. Let $\left(u_{0}, v_{0}, w_{0}\right) \neq(0,0,0)$ and $(u, v, w)$ be a solution of (1.1)-(1.2). Then we can choose $\tau=\tau\left(u_{0}, v_{0}, w_{0}\right)$ and constants $c, a>0$ such that $\min (u(\tau), v(\tau), w(\tau)) \geq c e^{-a|x|^{2}}$.

Proof. We use the same argument as in $[\mathrm{EL}]$ or $[\mathrm{EH}]$. Let, for instance, $u_{0} \neq 0$. We can assume that for some $R>0$,

$$
\nu=\inf \left\{u_{0}(x):|x|<R\right\}>0 .
$$

From $(2.1)_{1}$ we have

$$
u(t) \geq S(t) u_{0} \geq \nu(4 \pi t)^{-N / 2} \exp \left(-\frac{|x|^{2}}{4 t}\right) \int_{|y| \leq R} \exp \left(-\frac{|y|^{2}}{4 t}\right) d y .
$$

Defining

$$
\begin{gathered}
\bar{u}(t)=u\left(t+\tau_{0}\right) \quad \text { for some } \tau_{0}>0 \\
\alpha_{1}=\frac{1}{4 \tau_{0}}, \quad c_{1}=\nu\left(4 \pi \tau_{0}\right)^{N / 2} \int_{|y| \leq R} \exp \left(-\frac{|y|^{2}}{4 \tau_{0}}\right) d y
\end{gathered}
$$

we have

$$
\bar{u}(0)=u\left(\tau_{0}\right)>c_{1} \exp \left(-\alpha_{1}|x|^{2}\right) .
$$

In the same way we obtain

$$
\bar{v}(0)>c_{2} \exp \left(-\alpha_{2}|x|^{2}\right), \quad \bar{w}(0)>c_{3} \exp \left(-\alpha_{3}|x|^{2}\right) .
$$

Finally, we have to choose $\alpha$ and $c$ suitable for $u, v, w$ to ensure

$$
\left(u\left(x, \tau_{0}\right), v\left(x, \tau_{0}\right), w\left(x, \tau_{0}\right)\right)^{t}>c \exp \left(-\alpha|x|^{2}\right)(1,1,1)^{t}
$$

and this concludes the proof.

3. Global existence. In this section we prove Theorem 1 , considering separately the cases $\operatorname{det}(A-I)=0$ and $\operatorname{det}(A-I)<0$.

(a) $\operatorname{det}(A-I)=0$ (by $(1.5)$, this is equivalent to $p q r=1$ ). We want to find a global supersolution to system (1.1)-(1.2) of the form

$$
\left(\begin{array}{c}
\bar{u} \\
\bar{v} \\
\bar{w}
\end{array}\right)=\left(\begin{array}{c}
A e^{\alpha_{1} t} \\
B e^{\beta_{1} t} \\
C e^{\gamma_{1} t}
\end{array}\right)
$$

where, for given $u_{0}, v_{0}, w_{0}$, we choose $A, B, C$ so large that $A \geq\left\|u_{0}\right\|_{L^{\infty}}$, $B \geq\left\|v_{0}\right\|_{L^{\infty}}$ and $C \geq\left\|w_{0}\right\|_{L^{\infty}}$. Let $\alpha_{1}, \beta_{1}, \gamma_{1}$ be positive constants such that

$$
\bar{u}_{t}-\Delta \bar{u} \geq \bar{v}^{p}, \quad \bar{v}_{t}-\Delta \bar{v} \geq \bar{w}^{q}, \quad \bar{w}_{t}-\Delta \bar{w} \geq \bar{u}^{r},
$$


for all $x \in \mathbb{R}^{N}$ and $t>0$. Then (3.1) satisfies (3.2) for all $t>0$ if

$$
\begin{aligned}
& \alpha_{1}>A^{-1} B^{p} \exp \left(\left(p \beta_{1}-\alpha_{1}\right) t\right), \\
& \beta_{1}>B^{-1} C^{q} \exp \left(\left(q \gamma_{1}-\beta_{1}\right) t\right), \\
& \gamma_{1}>C^{-1} A^{r} \exp \left(\left(r \alpha_{1}-\gamma_{1}\right) t\right) .
\end{aligned}
$$

If we take $\beta_{1}=\alpha_{1} / p$ and $\gamma_{1}=\alpha_{1} /(p q)=r \alpha_{1}$ (the last equality follows from $p q r=1)$, then (3.2) holds for $\alpha_{1}$ large enough.

(b) $\operatorname{det}(A-I)<0$ (by $(1.5)$, that means $p q r<1$ ). We are looking for a global supersolution of the form

$$
\left(\begin{array}{c}
\bar{u} \\
\bar{v} \\
\bar{w}
\end{array}\right)=\left(\begin{array}{l}
A\left(t+t_{0}\right)^{\alpha_{1}} \\
B\left(t+t_{0}\right)^{\beta_{1}} \\
C\left(t+t_{0}\right)^{\gamma_{1}}
\end{array}\right),
$$

for some positive constants $A, B, C, \alpha_{1}, \beta_{1}, \gamma_{1}$ such that the inequalities (3.2) with $(\bar{u}, \bar{v}, \bar{w})$ given by (3.4) hold for all $x \in \mathbb{R}^{N}$ and $t>0$. We have to choose $t_{0}$ sufficiently large to satisfy

$$
\bar{u}(x, 0), \bar{v}(x, 0), \bar{w}(x, 0)) \geq\left(u_{0}, v_{0}, w_{0}\right)
$$

for $x \in \mathbb{R}^{N}$.

Substituting (3.4) into (3.2) we obtain the following conditions:

$$
\alpha_{1}-p \beta_{1} \geq 1, \quad \beta_{1}-q \gamma_{1} \geq 1, \quad \gamma_{1}-r \alpha_{1} \geq 1 .
$$

Let us remark that (3.6) has the form $(A-I)\left(-\alpha_{1},-\beta_{1},-\gamma_{1}\right)^{t} \geq(1,1,1)^{t}$ with $A$ given by (1.3). Set $\left(\alpha_{1}, \beta_{1}, \gamma_{1}\right)=-(\alpha, \beta, \gamma)$ for $\alpha, \beta, \gamma$ defined by (1.4). Since $p q r<1$ we see that $\left(\alpha_{1}, \beta_{1}, \gamma_{1}\right)$ are positive. Thus, (3.4) satisfies (3.2) and (3.5) provided that

$$
A \alpha_{1} \geq B^{p}, \quad B \beta_{1} \geq C^{q}, \quad C \gamma_{1} \geq A^{r},
$$

and $t_{0}$ is large enough. Then every nonnegative solution of (1.1)-(1.2) with bounded initial values is global.

4. Blow up (proof of Theorem 2). Without loss of generality we assume henceforth $r \leq q \leq p$. Thus, by (1.4), $\max (\alpha, \beta, \gamma)=\alpha$ for $p q r>1$.

LEMma 4.1. Let $(u(t), v(t), w(t))$ be a bounded solution of (1.1)-(1.2) in some strip $S_{T}$ with $0<T \leq \infty$. Let $p q r \geq 1$ and $r \geq 1$. Then there exists a positive constant $C$ such that

$$
t^{\alpha}\left\|S(t) u_{0}\right\|_{\infty} \leq C, \quad t \in[0, T)
$$

where $C$ depends on $p, q, r$ only and $\alpha$ is given by $(1.4)_{1}$. 
Proof. Using $(2.1)_{1}$ in $(2.1)_{3}$ we get

$$
w(t) \geq \int_{0}^{t} S(t-s)\left(S(s) u_{0}\right)^{r} d s
$$

and by the Jensen inequality for $r \geq 1$,

$$
w(t) \geq \int_{0}^{t}\left(S(t-s) S(s) u_{0}\right)^{r} d s=\int_{0}^{t}\left(S(t) u_{0}\right)^{r} d s=t\left(S(t) u_{0}\right)^{r} .
$$

We substitute (4.2) in $(2.1)_{2}$ (ignoring the first term on the right-hand side) and by the Jensen inequality we obtain

$$
\begin{aligned}
v(t) & \geq \int_{0}^{t} S(t-s)\left(s\left(S(s) u_{0}\right)^{r}\right)^{q} d s \geq \int_{0}^{t} s^{q}\left(S(t) u_{0}\right)^{r q} d s \\
& \geq \frac{1}{q+1}\left(S(t) u_{0}\right)^{r q} t^{q+1} .
\end{aligned}
$$

Using (4.3) in (2.1) 1 we can write

$$
\begin{aligned}
u(t) & \geq S(t-s)\left[\frac{1}{q+1}\left(S(s) u_{0}\right)^{r q} s^{q+1}\right]^{p} d s \\
& \geq\left(\frac{1}{q+1}\right)^{p}\left(S(t) u_{0}\right)^{p q r} \frac{t^{p(q+1)+1}}{p(q+1)+1} .
\end{aligned}
$$

Using again the lower bound (4.4) in $(2.1)_{3}$ gives

$$
w(t) \geq\left(\frac{1}{q+1}\right)^{r p}\left(S(t) u_{0}\right)^{p q r^{2}} \frac{1}{(p(q+1)+1)^{r}} \cdot \frac{t^{r p(q+1)+r+1}}{r p q+r p+r+1} .
$$

Continuing this procedure gives

$$
\begin{aligned}
v(t) \geq & \frac{1}{(q+1)^{r p q}} \cdot \frac{1}{(p(q+1)+1)^{r q}} \cdot \frac{1}{(r p(q+1)+r+1)^{q}} \\
& \times \frac{t^{(q+1)(r p q+1)+r q}\left(S(t) u_{0}\right)^{p q^{2} r^{2}}}{(q+1)(r p q+1)+r q} \\
u(t) \geq & \frac{1}{(q+1)^{r p^{2} q^{2}}} \cdot \frac{1}{(p q+p+1)^{r p q}} \cdot \frac{1}{(r p q+r p+r+1)^{p q}} \\
& \times \frac{1}{((q+1)(r p q+1)+r q)^{p}} \cdot \frac{1}{(r p q+1)(p(q+1)+1)} \\
& \times\left(S(t) u_{0}\right)^{p^{2} q^{2} r^{2}} t^{(r p q+1)(p q+p+1)} .
\end{aligned}
$$

Iterating this scheme, we obtain, using (1.4),

$$
u(t) \geq A_{k} B_{k} C_{k}\left(S(t) u_{0}\right)^{(p q r)^{k}} t^{\alpha \delta\left(1+r p q+\ldots+(r p q)^{k-1}\right)},
$$


where

$$
\begin{aligned}
A_{k}= & \frac{1}{(\alpha \delta)^{(r p q)^{k-1}}}\left[\frac{1}{(r p q+1) \alpha \delta}\right]^{(r p q)^{k-2}} \\
& \times\left[\frac{1}{\left((r p q)^{2}+r p q+1\right) \alpha \delta}\right]^{(r p q)^{k-3}} \\
& \times \ldots \times \frac{1}{\left((r p q)^{k-1}+\ldots+r p q+1\right) \alpha \delta}, \\
B_{k}= & \frac{1}{(q+1)^{p(r p q)^{k-1}}} \cdot \frac{1}{(r \alpha \delta+1)^{\frac{1}{r}(p q r)^{k-1}}} \\
& \times \frac{1}{(r \alpha \delta(r p q+1)+1)^{\frac{1}{r}(r p q)^{k-2}}} \\
& \times \frac{1}{\left(r \alpha \delta\left(1+r p q+\ldots+(r p q)^{k-2}\right)+1\right)^{p q}}, \\
C_{k}= & \frac{1}{(r q \alpha \delta+q+1)^{\frac{1}{r q}(r p q)^{k-1}}} \\
& \times \frac{1}{(r q \alpha \delta(r p q+1)+q+1)^{\frac{1}{r q}(r p q)^{k-2}}} \\
& \times \ldots \times \frac{1}{\left(r q \alpha \delta\left(1+p q r+\ldots+(p q r)^{k-2}\right)+q+1\right)^{p}} .
\end{aligned}
$$

Setting $p q r=z$ we can rewrite $(4.8)_{1}$ as

$$
A_{k}=\frac{1}{(\alpha \delta)^{\frac{z^{k-1}}{z-1}}}\left(\frac{1}{1+z}\right)^{z^{k-2}}\left(\frac{1}{1+z+z^{2}}\right)^{z^{k-3}} \ldots \frac{1}{1+z+\ldots+z^{k-1}}
$$

so

$$
A_{k}=\frac{1}{(\alpha \delta)^{\frac{z^{k}-1}{z-1}}} \prod_{j=1}^{k-1}\left(\frac{z-1}{z^{j+1}-1}\right)^{z^{k-j-1}} .
$$

Using $\alpha \delta\left(1+z+\ldots+z^{j}\right)>1+q$ for $j \geq 0$ we can estimate:

$$
\begin{aligned}
B_{k} \geq & \frac{1}{(q+1)^{p z^{k-1}}} \cdot \frac{1}{[(r+1) \alpha \delta]^{\frac{1}{r} z^{k-1}}} \cdot \frac{1}{[(r+1) \alpha \delta(1+z)]^{\frac{1}{r} z^{k-2}}} \\
& \times \ldots \times \frac{1}{\left[(r+1) \alpha \delta\left(1+z+\ldots+z^{k-2}\right)\right]^{p q}} \\
= & \frac{1}{(q+1)^{p z^{k-1}}} \cdot \frac{1}{[(r+1) \alpha \delta]^{\frac{1}{r} \cdot \frac{z^{k-z}}{z-1}}}\left[\prod_{j=1}^{k-2}\left(\frac{z-1}{z^{j+1}-1}\right)^{z^{k-j-1}}\right]^{\frac{1}{r}},
\end{aligned}
$$




$$
\begin{aligned}
C_{k} \geq & \frac{1}{[(r q+1) \alpha \delta]^{\frac{1}{r q}} z^{k-1}} \cdot \frac{1}{[(r q+1) \alpha \delta(z+1)]^{\frac{1}{r q} z^{k-2}}} \\
& \times \ldots \times \frac{1}{\left[(r q+1) \alpha \delta\left(1+z+\ldots+z^{k-2}\right)\right]^{p}} \\
= & \frac{1}{[(r q+1) \alpha \delta]^{\frac{1}{r q}} \cdot \frac{z^{k}-z}{z-1}}\left[\prod_{j=1}^{k-2}\left(\frac{z-1}{z^{j+1}-1}\right)^{z^{k-j-1}}\right]^{\frac{1}{r q}} .
\end{aligned}
$$

Substituting (4.9)-(4.11) into (4.7) we get

$$
\begin{aligned}
u(t) \geq & \left(S(t) u_{0}\right)^{z^{k}} t^{\alpha \delta \frac{z^{k}-1}{z-1}} \frac{1}{(q+1)^{p z^{k-1}}} \cdot \frac{1}{(r+1)^{\frac{1}{r} \cdot \frac{z^{k}-z}{z-1}}} \\
& \times \frac{1}{(r q+1)^{\frac{1}{r q} \cdot \frac{z^{k}-z}{z-1}}} \cdot \frac{1}{(\alpha \delta)^{\frac{z^{k}-1}{z-1}\left(1+\frac{1}{r}+\frac{1}{r q}\right)-\frac{1}{r}\left(1+\frac{1}{q}\right)}} \\
& \times\left[\prod_{j=1}^{k-2}\left(\frac{z-1}{z^{j+1}-1}\right)^{z^{k-j-1}}\right]^{1+\frac{1}{r}+\frac{1}{r q}} \frac{z-1}{z^{k}-1} .
\end{aligned}
$$

We infer that

$$
\begin{aligned}
t^{\alpha \delta \frac{z^{k}-1}{z^{k}(z-1)}} S(t) u_{0} \leq & (q+1)^{\frac{p}{z}}(r+1)^{\frac{1}{r z^{k}} \cdot \frac{z^{k}-z}{z-1}} \\
& \times(r q+1)^{\frac{1}{r q} \cdot \frac{z^{k}-z}{z^{k}(z-1)}}(\alpha \delta)^{\frac{1}{z^{k}}\left[\frac{z^{k}-1}{z-1}\left(1+\frac{1}{r}+\frac{1}{r q}\right)-\frac{1}{r}\left(1+\frac{1}{q}\right)\right]} \\
& \times\left[\prod_{j=1}^{k-2}\left(\frac{z-1}{z^{j+1}-1}\right)^{z^{j+1}}\right]^{1+\frac{1}{r}+\frac{1}{r q}}\left(\frac{z^{k}-1}{z-1}\right)^{1 / z^{k}}\|u(t)\|_{\infty}^{1 / z^{k}} .
\end{aligned}
$$

Letting $k \rightarrow \infty$ and using $\|u(t)\|_{\infty}<\infty$ we obtain in the limit

$$
t^{\alpha}\left\|S(t) u_{0}\right\|_{\infty} \leq c<\infty
$$

where $c=c(p, q, r)$ only.

Lemma 4.2. Assume that $p q r>1, p>1$ and $r \leq q<1$. Let $(u(t), v(t)$, $w(t))$ be as in Lemma 4.1. Then there exists a constant $C$ such that

$$
t^{r q \alpha}\left\|S(t) u_{0}^{r q}\right\|_{\infty} \leq C \quad \text { and } \quad C=C(p, q, r) .
$$

Proof. By the Jensen inequality for $r<1$ we have

$$
w(t) \geq t S(t) u_{0}^{r} \quad \text { and } \quad v(t) \geq \frac{1}{q+1} S(t) u_{0}^{r q} t^{q+1} .
$$

Repeating the iteration as in Lemma 4.1 we obtain

$$
u(t) \geq A_{k} B_{k} C_{k}\left(S(t) u_{0}^{r q}\right)^{\frac{1}{r q}(p q r)^{k}} t^{\alpha \delta\left(1+\ldots+(p q r)^{k-1}\right)}
$$

and $A_{k}, B_{k}, C_{k}$ are given by (4.9)-(4.11). So we estimate as before and 
letting $k \rightarrow \infty$ we conclude that

$$
t^{r q \alpha}\left\|S(t) u_{0}^{r q}\right\|_{\infty} \leq C .
$$

We complete the result by considering the case $r<1<q$.

LEMMA 4.3. Let $p q r>1$ with $p>1$ and $r<1<q$, and let $u, v, w$ be as in Lemma 4.1. Then

$$
t^{r \alpha}\left\|S(t) u_{0}^{r}\right\|_{\infty} \leq C,
$$

where the constant $C$ depends on $p, q, r$ only.

P r o of. We argue as in the previous lemma, starting from $w(t) \geq t S(t) u_{0}^{r}$ and

$$
v(t) \geq \frac{1}{q+1}\left(S(t) u_{0}^{r}\right)^{q} t^{q+1}
$$

so

$$
u(t) \geq A_{k} B_{k} C_{k}\left(S(t) u_{0}^{r}\right)^{\frac{1}{r}(p q r)^{k}} t^{\alpha \delta\left(1+p q r+\ldots+(p q r)^{k-1}\right)}
$$

and we get (4.14) as before.

LEMMA 4.4. Let $p q r>1$, and let $(u(t), v(t), w(t))$ be a bounded solution of (1.1)-(1.2) (as in Lemma 4.1). Then we can find a constant $C>0$, $C=C(p, q, r)$, such that for $t>0$,

$$
\begin{array}{ll}
t^{\alpha}\|S(t) u(t)\|_{\infty} \leq C<\infty & \text { if } 1<r \leq q \leq p, \\
t^{r q \alpha}\left\|S(t) u(t)^{r q}\right\|_{\infty} \leq C<\infty & \text { if } r \leq q<1<p, \\
t^{r \alpha}\left\|S(t) u(t)^{r}\right\|_{\infty} \leq C<\infty & \text { if } r<1<q \leq p .
\end{array}
$$

Proof. For $\tau, t \geq 0$ we can rewrite $(2.1)_{3}$ in the form

$$
\begin{aligned}
w(t+\tau) & =S(t+\tau) u_{0}+\int_{0}^{t+\tau} S(t+\tau-s) u(s)^{r} d s \\
& =S(t) u(\tau)+\int_{0}^{t} S(t-s) u(\tau+s)^{r} d s
\end{aligned}
$$

and similarly for $v$ and $u$. Hence we can replace $u_{0}$ by $u(\tau)$ in (4.1), (4.12) and (4.14); setting $t=\tau$, we get the conclusion.

Lemma 4.5. Suppose that $\alpha \geq N / 2$ and $p q r>1$. Then every nontrivial solution of (1.1)-(1.2) blows up in finite time.

Proof. Assume that there exists a bounded solution of (1.1)-(1.2) with $\left(u_{0}, v_{0}, w_{0}\right)>(0,0,0)$ and $\alpha \geq N / 2, p q r>1$. By Lemma 2.1 we can find $c, a>0$ such that 


$$
u_{0}(x) \geq c e^{-a|x|^{2}} .
$$

We will also use the following equality:

$$
S(t) e^{-a|x|^{2}}=(1+4 a t)^{-N / 2} \exp \left(\frac{-a|x|^{2}}{1+4 a t}\right) .
$$

First, we consider the case $0<r<1 \leq q<p$. Using (4.16) in (4.17) we get

$$
u(t) \geq S(t) u_{0} \geq c(1+4 a t)^{-N / 2} \exp \left(\frac{-a|x|^{2}}{1+4 a t}\right) .
$$

Now, from $(2.1)_{3}$, the last inequality and (4.17) we obtain

$$
\begin{aligned}
w(t) \geq & \int_{0}^{t} S(t-s) u(s)^{r} d s \\
\geq & c^{r} \int_{0}^{t} S(t-s)(1+4 a s)^{-N r / 2} \exp \left(\frac{-a r|x|^{2}}{1+4 a s}\right) d s \\
= & c^{r} \int_{0}^{t}(1+4 a s)^{-N r / 2}\left(1+\frac{4 a r}{1+4 a s}(t-s)\right)^{-N / 2} \\
& \times \exp \left(\frac{-a r|x|^{2}}{1+4 a s+4 a r(t-s)}\right) d s \\
= & c^{r} \int_{0}^{t}(1+4 a s)^{-N(r-1) / 2}(1+4 a s+4 a r(t-s))^{-N / 2} \\
& \times \exp \left(\frac{-a r|x|^{2}}{1+4 a s+4 a r(t-s)}\right) d s .
\end{aligned}
$$

We note that $f(s)=1+4 a s+4 a r(t-s)$ is increasing (because $f^{\prime}(s)=$ $4 a(1-r)>0)$ so that

$$
w(t) \geq c^{r}(1+4 a t)^{-N / 2} \exp \left(\frac{-a r|x|^{2}}{1+4 a r t}\right) \int_{0}^{t}(1+4 a s)^{-N(r-1) / 2} d s .
$$

Integrating, we obtain

$$
\geq \frac{w(t)}{4 a(1-N(r-1) / 2)}(1+4 a t)^{-N / 2} \exp \left(\frac{-a r|x|^{2}}{1+4 a r t}\right)(4 a t)^{1-N(r-1) / 2} .
$$

Now, we use (4.18) in $(2.1)_{2}$ to get (by (4.17)) 


$$
\begin{aligned}
v(t) \geq & \frac{c^{r q}}{[4 a(1-N(r-1) / 2)]^{q}} \int_{0}^{t}(1+4 a s)^{-N q / 2}(4 a s)^{q(1-N(r-1) / 2)} \\
& \times S(t-s) \exp \left(\frac{-a r q|x|^{2}}{1+4 a r s}\right) d s \\
= & \frac{c^{r q}}{[4 a(1-N(r-1) / 2)]^{q}} \\
& \times \int_{0}^{t}(1+4 a s)^{-N q / 2}(1+4 a r s)^{N / 2}(4 a r s)^{q(1-N(r-1) / 2)} \\
& \times(1+4 a r s+4 a r q(t-s))^{-N / 2} \exp \left(\frac{-\operatorname{arq}|x|^{2}}{1+4 a r+4 a r q(t-s)}\right) d s .
\end{aligned}
$$

Consider $g(s)=1+4 \operatorname{ar} s+4 \operatorname{ar} q(t-s)$; as $g^{\prime}(s)=4 \operatorname{ar}(1-q)<0$ we deduce that

$$
\begin{aligned}
v(t) \geq & \frac{c^{r q}}{[4 a(1-N(r-1) / 2)]^{q}}(1+4 a r q t)^{-N / 2} \exp \left(\frac{-a r q|x|^{2}}{1+4 a r t}\right) \\
& \times \int_{0}^{t}(1+4 a s)^{-N q / 2}(1+4 a r s)^{N / 2}(4 a s)^{q(1-N(r-1) / 2)} d s .
\end{aligned}
$$

To integrate, let us remark that $(1+4 a r s)^{N / 2} \geq r^{N / 2}(1+4 a s)^{N / 2}$ and $4 a s>\frac{1}{2}(1+4 a s)$ for $s>1 /(4 a)$. Denoting by $c_{1}$ the new constant such that

$$
c_{1}:=\frac{c^{r q}}{[4 a(1-N(r-1) / 2)]^{q}} r^{N / 2}\left(\frac{1}{2}\right)^{q(1-N(r-1) / 2)},
$$

we have

$$
v(t) \geq c_{1}(1+4 a r q t)^{-N / 2} \exp \left(\frac{-a r q|x|^{2}}{1+4 a r t}\right) \int_{0}^{t}(1+4 a s)^{q-N(q r-1) / 2} d s
$$

and finally

$$
v(t) \geq c(1+4 a r q t)^{-N / 2}(4 a t)^{1+q-N(q r-1) / 2} \exp \left(\frac{-a r q|x|^{2}}{1+4 a r t}\right),
$$

where $c=c(p, q, r, N / 2, a)$ is a constant.

We need a lower bound for $u(t)$, so we substitute (4.19) into $(2.1)_{1}$ to get

$$
\begin{aligned}
u(t) \geq & c^{p} \int_{0}^{t}(1+4 a r q s)^{-N p / 2}(4 a s)^{p(1+q-N(q r-1) / 2)} \\
& \times S(t-s) \exp \left(\frac{-a p q r|x|^{2}}{1+4 a r s}\right) d s \\
= & c^{p} \int_{0}^{t}(1+4 a r q s)^{-N p / 2}(4 a s)^{p(1+q-N(q r-1) / 2)}
\end{aligned}
$$




$$
\begin{aligned}
& \times\left(1+\frac{\operatorname{4arpq}(t-s)}{1+4 a r s}\right)^{-N / 2} \\
& \times \exp \left(\frac{-a p q r|x|^{2}}{1+4 a r s+4 a r p q(t-s)}\right) d s .
\end{aligned}
$$

The last equality follows by (4.17). Now, consider $h(s)=1+4$ ars + $4 \operatorname{arpq}(t-s)$; note that $h^{\prime}(s)=4 \operatorname{ar}(1-p q)<0$ so as before we get

$$
\begin{aligned}
u(t) \geq & c^{p}(1+4 a r p q t)^{-N / 2} \exp \left(\frac{-a p q r|x|^{2}}{1+4 a r t}\right) \\
& \times \int_{0}^{t}(1+4 a r q s)^{-N p / 2}(4 a s)^{p(1+q-N(q r-1) / 2)}(1+4 a r s)^{N / 2} d s .
\end{aligned}
$$

Using again

$$
\begin{gathered}
4 a t>\frac{1}{2}(1+4 a t) \quad \text { for } t>1 /(4 a), \\
(1+4 a r s)^{N / 2} \geq r^{N / 2}(1+4 a s)^{N / 2} \quad \text { for } r<1,
\end{gathered}
$$

and noting that

$$
(1+4 a r q t)^{-N / 2} \geq(1+4 a p q r t)^{-N / 2} \geq(p q r)^{-N / 2}(1+4 a t)^{-N / 2}
$$

holds since $p>1$ and $p q r>1$, we obtain from (4.20), for $t>1 /(4 a)$,

$$
u(t) \geq c(1+4 a t)^{-N / 2} \exp \left(\frac{-a p q r|x|^{2}}{1+4 a r t}\right) \int_{1 /(4 a)}^{t}(1+4 a s)^{\varrho} d s,
$$

where

$$
\begin{aligned}
\varrho & =-N p / 2+p(1+q-N(q r-1) / 2)+N / 2 \\
& =p+p q-N(p q r-1) / 2 \geq-1
\end{aligned}
$$

by the assumption that $\alpha \geq N / 2$.

So we infer that

$$
u(t) \geq c(1+4 a t)^{-N / 2} \exp \left(\frac{-a p q r|x|^{2}}{1+4 a r t}\right) \log \left(\frac{4 a t+1}{2}\right)
$$

for $t>1 /(4 a)$.

It now follows by (4.17) that

$$
\begin{aligned}
S(t) u(t)^{r} \geq & c(1+4 a t)^{-N r / 2} \exp \left(\frac{-a p q r^{2}|x|^{2}}{1+4 a r t}\right) \\
& \times S(t)\left[\log \left(\frac{1+4 a t}{2}\right)\right]^{r} \\
= & c(1+4 a t)^{-N r / 2}(1+4 a r(1+r p q) t)^{-N / 2}(1+4 a r t)^{N / 2} \\
& \times\left[\log \left(\frac{1+4 a t}{2}\right)\right]^{r} \exp \left(\frac{-a r^{2} p q|x|^{2}}{1+4 a r(1+r p q) t}\right)
\end{aligned}
$$




$$
\begin{aligned}
\geq & c(1+4 a t)^{-N r / 2}\left(\frac{1+4 a r t}{(1+4 a r t)(1+p q r)}\right)^{N / 2} \\
& \times\left[\log \left(\frac{1+4 a t}{2}\right)\right]^{r} \exp \left(\frac{-a r^{2} p q|x|^{2}}{1+4 a r(1+r p q) t}\right) .
\end{aligned}
$$

Putting $x=0$ in $(4.22)$ we get

$$
(1+4 a t)^{N r / 2} S(t) u(t, 0)^{r} \geq \frac{c}{(1+p q r)^{N / 2}}\left[\log \left(\frac{1+4 a t}{2}\right)\right]^{r}
$$

and therefore, for $t>\max (1,1 /(4 a))$ and since $\alpha \geq N / 2$,

$$
t^{r \alpha} S(t) u(t, 0)^{r} \geq c\left[\log \left(\frac{1+4 a t}{2}\right)\right]^{r} .
$$

It remains to notice that as $t \rightarrow \infty$, the right-hand side of (4.23) diverges, and so does the left-hand side. But this contradicts $(4.15)_{3}$. Thus, $u(t)$ must become unbounded, and by $(2.1), v(t)$ and $w(t)$ also blow up in finite time.

Now, we discuss the remaining cases.

In the case $0<r \leq q<1<p$ we argue as before to get, instead of (4.21),

$$
u(t) \geq c(1+4 a t)^{-N / 2} \exp \left(\frac{-\operatorname{arpq}|x|^{2}}{1+4 a r q t}\right) \log \left(\frac{4 a t+1}{2}\right)
$$

and

$$
\begin{aligned}
S(t) u(t)^{r q} \geq & c(1+4 a t)^{-N q r / 2} \exp \left(\frac{-a r^{2} q^{2} p|x|^{2}}{1+4 a r q t}\right) \\
& \times S(t)\left[\log \left(\frac{1+4 a t}{2}\right)\right]^{q r} \\
= & c(1+4 a t)^{-N q r / 2}\left(\frac{1+4 a r q t}{1+4 a r q(1+p q r) t}\right)^{N / 2} \\
& \times \exp \left(\frac{-a r^{2} q^{2} p|x|^{2}}{1+4 a r q(1+p q r) t}\right)\left[\log \left(\frac{1+4 a t}{2}\right)\right]^{q r} .
\end{aligned}
$$

Thus, for $x=0$,

$$
S(t) u(t, 0)^{q r}(1+4 a t)^{q r N / 2} \geq c\left[\log \left(\frac{1+4 a t}{2}\right)\right]^{q r},
$$

which implies, for $t>\max (1,1 /(4 a))$, as $\alpha \geq N / 2$,

$$
t^{q r \alpha} S(t) u(t, 0)^{q r} \geq c\left[\log \left(\frac{1+4 a t}{2}\right)\right]^{q r} .
$$

Now, we see that (4.24) is incompatible with $(4.15)_{2}$ for $t$ large enough. 
Finally, we consider the case $1<r \leq q \leq p$. Then instead of (4.21) we infer that

$$
u(t) \geq c(1+4 a t)^{-N / 2} \exp \left(\frac{-a r p q|x|^{2}}{1+4 a t}\right) \log \left(\frac{4 a t+1}{2}\right),
$$

whence

$$
S(t) u(t)(1+4 a t)^{N / 2} \geq c \exp \left(\frac{-a r p q|x|^{2}}{1+4 a(1+p q r) t}\right) \log \left(\frac{4 a t+1}{2}\right) .
$$

Setting $x=0$ and using $\alpha \geq N / 2$, we have

$$
t^{\alpha} S(t) u(t, 0) \geq c \log \left(\frac{4 a t+1}{2}\right)
$$

which contradicts $(4.15)_{1}$ for $t$ large.

Thus, in each case, we have a contradiction and the proof is complete.

\section{References}

[AHV] D. Andreucci, M. A. Herrero and J. J. L. Velázquez, Liouville theorems and blow up behaviour in semilinear reaction diffusion systems, Ann. Inst. $\mathrm{H}$. Poincaré 14 (1997), 1-53.

[EH] M. Escobedo and M. A. Herrero, Boundedness and blow up for a semilinear reaction-diffusion system, J. Differential Equations 89 (1991), 176-202.

[EL] M. Escobedo and H. A. Levine, Critical blow up and global existence numbers for a weakly coupled system of reaction-diffusion equations, Arch. Rational Mech. Anal. 129 (1995), 47-100.

[F1] H. Fujita, On the blowing up of solutions of the Cauchy problem for $u_{t}=$ $\nabla u+u^{1+\alpha}$, J. Fac. Sci. Univ. Tokyo Sect. IA Math. 13 (1966), 109-124.

[F2] - On some nonexistence and nonuniqueness theorems for nonlinear parabolic equations, in: Proc. Sympos. Pure Math. 18, Amer. Math. Soc., 1970, 105-113.

Joanna Renclawowicz

Institute of Mathematics

Polish Academy of Sciences

Śniadeckich 8

00-950 Warszawa, Poland

E-mail: jr@impan.gov.pl 